\title{
Challenges to Abdominal and Cardiac Surgeons: Emergency Splenectomy after LVAD Implantation-A Case Report
}

\author{
Dusko Terzic, MD, ${ }^{1,}$ Emilija Nestorovic, $\mathrm{MD}, \mathrm{PhD},{ }^{1}$ Miljan Ceranic, $\mathrm{MD}, \mathrm{PhD},{ }^{2,3}$ \\ Aleksandar Mikic, MD, PhD, ${ }^{1,3}$ Vladimir Milicevic, MD, ${ }^{1}$ and Svetozar Putnik, $\mathrm{MD}, \mathrm{PhD}^{1,3}$ \\ ${ }^{1}$ Clinic for Cardiac Surgery, Clinical Center of Serbia, and ${ }^{2}$ Clinic for Digestive Surgery, Clinical Center of Serbia, Belgrade, Serbia; \\ ${ }^{3}$ University of Belgrade Faculty of Medicine, Belgrade, Serbia
}

\section{ABSTRACT}

The paper presents collaboration of an abdominal surgeon and heart team in deciding upon surgical management of a patient with the implanted left ventricular assist device, who has undergone emergency abdominal operation for spleen rupture. The paper focuses on the significance of prompt diagnostics, clinical challenges of hemodynamic and anticoagulant treatment, abdominal organ exposure along the placed left ventricular assist device driveline, identification of vascular structures in conditions of continuous blood flow, and reconstruction of the surgical wound in the driveline projection.

\section{INTRODUCTION}

Implantable mechanical circulatory devices, including the left ventricular assist device (LVAD), have emerged as a relevant option for improving the quality of life and survival in patients with the end-stage heart failure.Bridge to transplant, destination therapy, and bridge to recovery are the most common indications [Takeda 2014].

Technological progress of the structure and function of the LVAD device as well as refined clinical practice of implantation and postimplantation follow-up of patients has contributed to prolongation of lifespan in LVAD patients, and, accordingly, the need for noncardiac surgery has been continuously rising [Slaughter 2009].

\section{CASE REPORT}

We present a 28 -year-old male patient with the LVAD HeartMate II, as bridge to transplant, implanted because of end-stage heart failure caused by dilatative cardiomyopathy. The patient was urgently transported to our institution for abdominal pain and weakness. Accordingto an urgent

Received fuly 17, 2018; received in revised form September 10, 2018; accepted September 14, 2018.

Correspondence: Dusko Terzic, Koste Todorovica 8, 11000 Belgrade; 381668301966 (terzic.dusko@gmail.com). diagnostic algorithm, the spleen rupture and hematoperitoneum were confirmed. After the LVAD implantation 4 years ago, the patient has been completely rehabilitated from the cardiopulmonary aspect; the patient's life habits and postimplantation social status have changed to be in line with his age group, and 18 months from the intervention he got married and had a healthy child.

The patient reported that the pain appeared during lifting of an approximately $15-\mathrm{kg}$ object. On admission, he complained of an extensive abdominal pain, nausea, and vomiting. Abdominal MSCT revealed nonhomogeneous parenchyma of the total spleen, and a large amount of diffuse free fluid that was hyperdense and had high attenuation values corresponding to hemorrhagic content. Laboratory blood analyses showed a big fall of blood count parameters,with $\mathrm{Hb} 98 \mathrm{~g} / \mathrm{L}$ on admission and $78 \mathrm{~g} / \mathrm{L}$ upon the patient being transported to the operating room (Figure 1).

For earlier hospital stays for LVAD control, heparininduced thrombocytopenia had been confirmed, which was an additional problem for preparation of urgent abdominal operation given that warfarin could not be replaced by nonfractionated Farin. Inspection of LVAD recorded speed pump, pump flow, and pump power of $9200 \mathrm{rpm}, 4.5 \mathrm{~L} / \mathrm{min}$, and 5.2 watts, respectively.

The case was discussed at our multidisciplinary heart team and general surgery team meeting, and it was decided to carry out an immediate surgical intervention that would include a general surgeon, a cardiac surgeon, a cardiac anesthesiologist,a blood bank technology specialist, cardiologist, and an LVAD technician.

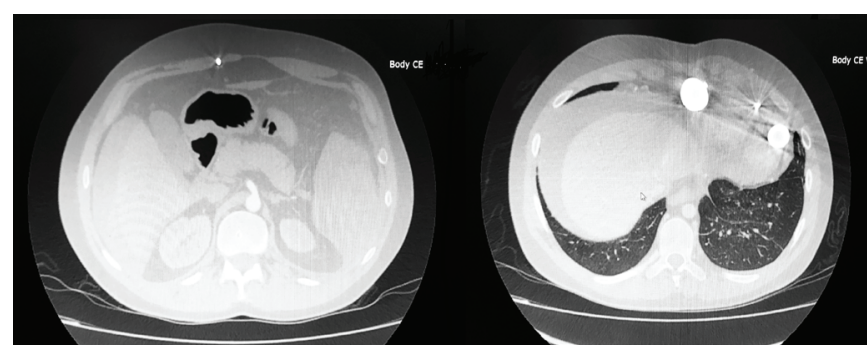

Figure 1.Nonhomogeneous parenchyma of the spleen, and a large amount of free fluid corresponding to hemorrhagic content. 


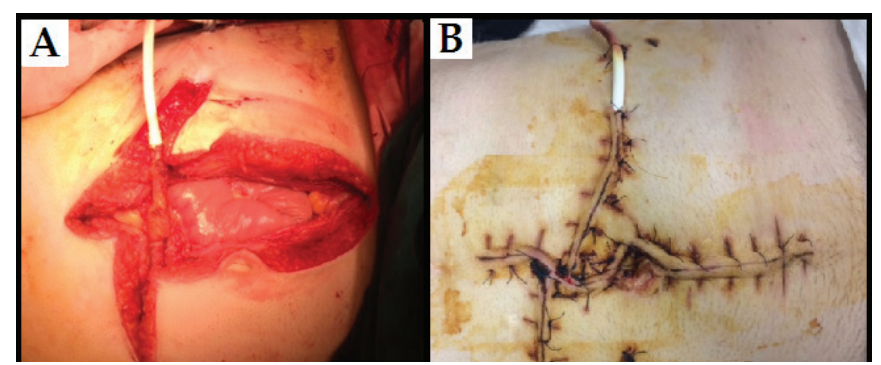

Figure 2. (A),Medial laparotomy was expanded by driveline mobilization using a horizontal cut in its projection. (B), Shape of the wound as a whole was like an irregular cross (shown is healing of this complex wound after 15 days)..

On account of active bleeding and volume loss, the pump speed, flow, and power were initially reduced before the operation to $9000 \mathrm{rpm}, 4.2 \mathrm{~L} / \mathrm{min}$, and 5.1 watts, respectively; thereupon, during the intervention, the speed was additionally lowered to $8800 \mathrm{rpm}$. INR value on admission was 4.4, which was corrected by applying prothrombin complex concentrate (PCC); and when the operation was completed, its value was figured at 1.74 .

The patient was immediately transported to the operating room and underwent surgery including medial laparotomy with releasing of the driveline from the curve in the abdominal right upper quadrant to the driveline exit site. Upon opening the peritoneum, about $2000 \mathrm{~mL}$ of blood and blood clots were evacuated. The spleen rupture was ascertained, and splenectomy with thorough hemostasis was performed. The surgical wound was reconstructed by using the interrupted sutures. After the operation, the pump speed, flow, and power were reset to $9200 \mathrm{rpm}, 4.8 \mathrm{~L} / \mathrm{min}$, and 6.2 watts, respectively.

Operative and postoperative courses were uneventful. Warfarin was reintroduced into therapy on the second postoperative day. The INR value on the first postoperative day was spontaneously maintained within the therapeutic range 2.2. After operation, the patient was fully rehabilitated. Wound dressing was done on a daily basis, and it healed by first intention. The sutures were removed in several phases, from days 20 to 30 after the operation (they are commonly removed from days 10 to 14 ), which we consider vital for safe healing of this complex wound (Figure 2B).

The patient was discharged onpostoperative day 32, was hemodynamically stable, and tolerated physical exercise quite well. At control visits, after one and 3 months, the surgical wound healed by first intention, and the driveline exit site manifested no signs of erythema, sensibility, or other signs of apparent infection. The LVAD parameters were regular. The patient has been listed in the heart transplant waiting list.

\section{DISCUSSION}

Emergency abdominal operations after LVAD implantation remain particularly challenging. The situations of the patients undergoing the abovementioned surgery are complex, and these interventions carry high postoperative morbidity and mortality. Therefore, it is necessary to provide a safe operating noncardiosurgical procedure and to have the collaboration of a general surgeon, a cardiac surgeon, a cardiologist, an anesthesiologist, and an LVAD coordinator.Allthe aforesaid implies that such procedures, if feasible during an emergency, should be carried out in institutions with established and wellorganized clinical services.

There are many clinical challenges in urgent abdominal operations of patients with the implanted LVAD. In a study by Nakamura et al, many of the patients at the time of admission received anticoagulation and/or antiaggregation therapy, so the control of the INR value during the intervention or immediately after the intervention is necessary [Nakamura 2017].

Given that patients with the implanted device for permanent circulatory support lack arterial pulse, an identification of abdominal arterial structures may be another challenge to the surgeon.

It is important to have clear insight into the direction of the driveline extension, the exit site of the cable from the mediastinum in the subxiphoid region, as well as the depth of the cable extension through the subcutaneous tissue all the way to its exit from the skin. Radiological images may secure clear vision of the pump position, its relation to adjacent structures, and the exit cable extension (driveline) [Mejia 2017] (Figures 3A and 3B).

Given that the driveline as a foreign body often needs repositioning, maximal aseptic measures are required. The incision should be created in the way to avoid possible driveline moving; but in conditions of urgent operation it is often necessary, for providing adequate exposure of the organs, to move the driveline from its original bearing. Upon completion of the abdominal operation, it is indispensable to make a new driveline bearing and leave a long distance from the driveline exit site to the pump pocket in order to prevent the infection [Trachtenberg 2015].

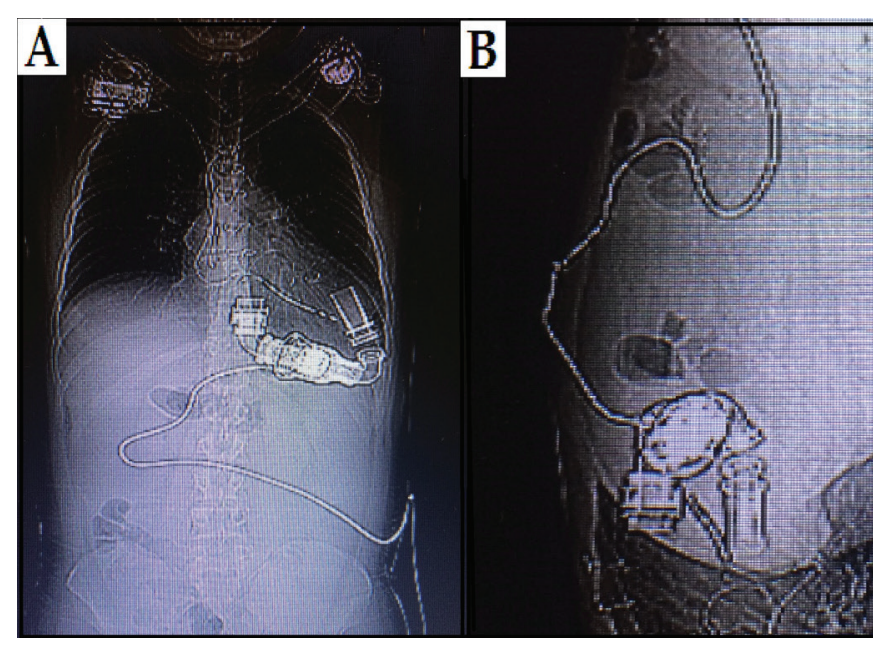

Figure 3. (A), Direction of the driveline extension in C-mode shape. (B), Driveline in the subxiphoid region and the depth of the cable extension through the subcutaneous tissue. 
In our patient, the INR value was corrected by PCC immediately before the intervention and during the operation itself. Considering that the position of the ruptured and enlarged spleen required a wide incision for adequate and safe approach, medial laparotomy was expanded by driveline mobilization using a horizontal cut in its projection, and therefore the shape of the wound as a whole was like an irregular cross (Figures $2 \mathrm{~A}$ and $2 \mathrm{~B}$ ). Upon splenectomy, the abdominal wall was reconstructed; and the driveline, because there were no signs of local infection, was moved back to its original bearing, anda new exit site was created.

\section{CONCLUSION}

Abdominal surgery in patients with the implanted LVAD should be considered carefully.Close collaboration with a heart-LVAD team and an abdominal surgeon is crucial for adequate and successful treatment of these patients. Driveline position in C-mode shape may require widened laparotomic incision, but it is not a contraindication for medial laparotomy.

\section{REFERENCES}

Takeda K, Takayama H, Kalesan B, et al. 2014. Long-term outcome of patients on continuous-flow left ventricularassist device support. J Thorac Cardiovasc Surg148:1606-14.

Slaughter MS, Rogers JG, Milano CA, et al. 2009. Advanced heart failure treated with continuous-flow left ventricular assist device. N Engl J Med361:2241-51.

Nakamura Y, Toda K, Nakamura T, et al. 2017. Curative surgery for gastric cancer in a patient with an implantable left ventricular assist device. J Artif Organs20:170-3.

Mejia JC, Dong M, Ojogho O. 2017. Pancreaticoduodenectomy in a patient with previous left ventricular assist device: a case report with specific emphasis on peri-operative logistics.J Surg Case Rep2017:rjx053.

Trachtenberg BH, Cordero-Reyes A, Elias B, Loebe M.2015. A review of infections in patients with left ventricular assist devices: prevention, diagnosis and management. Methodist Debakey Cardiovasc J11:28-32. 